\title{
On Neutrosophic Quadruple Hypervector Spaces
}

\author{
${ }^{1}$ M.A. Ibrahim, ${ }^{2}$ A.A.A. Agboola , ${ }^{3}$ E.O. Adeleke, ${ }^{4}$ S.A. Akinleye \\ $1,2,3,4$ Department of Mathematics, Federal University of Agriculture, Abeokuta, Nigeria. \\ muritalaibrahim40@gmail.com ${ }^{1}$,agboolaaaa@funaab.edu.ng ${ }^{2}$,yemi376@yahoo.com ${ }^{3}$, \\ sa_akinleye@yahoo.com ${ }^{4}$
}

\begin{abstract}
The objective of this paper is to study Neutrosophic Quadruple Hypervector Spaces and present some of their basic definitions and properties. This paper generalizes the concept of Neutrosophic Hypervector spaces by presenting their Neutrosophic Quadruple forms. Some notions such as Neutrosphic hypersubspaces, linear combination, linearly dependence and linearly independence are generalized. Some interesting results and examples to illustrate the new concepts introduced are presented.

Keywords: Neutrosophic Quadruple (NQ), Neutrosophic Quadruple set, NQ Hypervector spaces, Super strong NQ Hypervector spaces, strong NQ Hypervector spaces, Weak NQ Hypervector spaces, NQ field, Neutrosophic field, NQ Hypersubspaces, NQ bases.
\end{abstract}

\section{Introduction}

Neutrosophy is a new branch of philosophy that studies the origin, nature, and scope of neutralities, as well as their interactions with different ideational spectra. Neutrosophic set and neutrosophic logic were introduced in 1995 by Smarandache as generalizations of fuzzy set and respectively intuitionistic fuzzy logic. In neutrosophic logic, each proposition has a degree of truth $(T)$, a degree of indeterminacy $(I)$, and a degree of falsity $(F)$, where $T, I, F$ are standard or non-standard subsets of $]^{-} 0,1^{+}[$, see [27|28|29].

The notion of neutrosophic algebraic structures was introduced by Kandasamy and Smarandache in 2006, see 32.33 Since then, several researchers have studied the concepts and a great deal of literature has been produced. For example, Agboola and Akinleye introduced the concept of neutrosophic hypervector spaces in ${ }^{1}$ and in ${ }^{2}$ they studied neutrosophic vector spaces. In, $\frac{34}{\sqrt{3}}$ Vasantha K., Ilanthenral K. and Smarandache F. introduced for the first time the concept of neutrosophic qudruple vector spaces over the classical fields $\mathbb{R}, \mathbb{C}$ and $\mathbb{Z}_{p}$. A comprehensive review of neutrosophy, neutrosophic triplet set, neutrosophic quadruple set and neutrosophic algebraic structures can be found in [3||||||1|11|12|14|15|17|18||19|20|2|[22|30|31]$]$.

The concept of hyperstructure was first introduced by Marty ${ }^{16}$ in 1934 at the 8 th congress of Scandinavian Mathematicians and then he established the definition of hypergroup in 1935 to analyze its properties and applied them to groups of rational algebraic functions. M. Krasner ${ }^{13}$ introduced the notions of hyperring and hyperfield and use them as technical tools in the study of the approximation of valued fields. These concepts have been developed and generalized by many researchers.

The notion of hypervector spaces was introduced by M. Scafati Tallini24 in 1988. Hypervector spaces have been further expanded by other researchers. For more detailed information on hypervector spaces, the reader

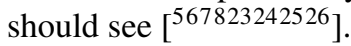

The present paper is concerned with introducing the concept of neutrosophic quadruple hypervector spaces. Some of their elementary properties are presented.

\section{Preliminaries}

In this section, some basic definitions and properties that will be useful in this work are given. 
Definition 2.1. A neutrosophic quadruple number is a number of the form $(a, b T, c I, d F)$ where $T, I, F$ have their usual neutrosophic logic meanings and $a, b, c, d \in \mathbb{R}$ or $\mathbb{C}$. The set $N Q$ defined by

$N Q=\{(a, b T, c I, d F): a, b, c, d \in \mathbb{R}$ or $\mathbb{C}\}$ is called neutrosophic quadruple set.

Definition 2.2. ${ }^{4}$ Suppose in an optimistic way we consider the prevalence order $T>I>F$. Then the combination of the usual Neutrosophic tools $T, I, F$ are :

$T I=I T=\max \{T, I\}=T$,

$T F=F T=\max \{T, F\}=T$,

$I F=F I=\max \{I, F\}=I$,

$T T=T^{2}=T$,

$I I=I^{2}=I$,

$F F=F^{2}=F$

Analogously, suppose in a pessimistic way we consider the prevalence order $T<I<F$. Then we have: $T I=I T=\max \{T, I\}=I$,

$T F=F T=\max \{T, F\}=F$,

$I F=F I=\max \{I, F\}=F$,

$T T=T^{2}=T$,

$I I=I^{2}=I$,

$F F=F^{2}=F$

We shall adopt the pessimistic way in this work.

The following operations are defined on $N Q$, for $x=(a, b T, c I, d F)$ and $y=(e, f T, g I, h F) \in N Q$ we have that

$$
\begin{gathered}
x+y=(a, b T, c I, d F)+(e, f T, g I, h F)=(a+e,(b+f) T,(c+g) I,(d+h) F) \text { and } \\
x-y=(a, b T, c I, d F)-(e, f T, g I, h F)=(a-e,(b-f) T,(c-g) I,(d-h) F) \text { are in NQ. }
\end{gathered}
$$

For $x=(a, b T, c I, d F) \in N Q$ and $k \in \mathbb{R}$ where $k$ is a scalar and $x$ is a vector in $N Q$.

$$
k . x=k .(a, b T, c I, d F)=(k a, k b T, k c I, k d F) \in N Q .
$$

If $x=0=(0,0,0,0) \in N Q$ usually termed as zero neutrosophic quadruple vector and for any scalar $k \in \mathbb{R}$ we have $k \cdot 0=0$.

Further

$$
(k+p) x=k x+p x, k(p x)=(k p) x, k(x+y)=k x+k y .
$$

for all $k, p \in \mathbb{R}$ and $x, y \in N Q .-x=(-a,-b T,-c I,-d F)$ which is in $N Q$.

Definition 2.3. Let $a=\left(a_{1}, a_{2} T, a_{3} I, a_{4} F\right), b=\left(b_{1}, b_{2} T, b_{3} I, b_{4} F\right) \in N Q$. Then

$$
\begin{aligned}
a \cdot b= & \left(a_{1}, a_{2} T, a_{3} I, a_{4} F\right) \cdot\left(b_{1}, b_{2} T, b_{3} I, b_{4} F\right) \\
= & \left(a_{1} b_{1},\left(a_{1} b_{2}+a_{2} b_{1}+a_{2} b_{2}\right) T,\left(a_{1} b_{3}+a_{2} b_{3}+a_{3} b_{1}+a_{3} b_{2}+a_{3} b_{3}\right) I,\right. \\
& \left.\left(a_{1} b_{4}+a_{2} b_{4}, a_{3} b_{4}+a_{4} b_{1}+a_{4} b_{2}+a_{4} b_{3}+a_{4} b_{4}\right) F\right) .
\end{aligned}
$$

Theorem 2.4. $4(N Q,+)$ is an abelian group.

Theorem 2.5. $4(N Q, \cdot)$ is a commutative monoid.

Theorem 2.6. $4(N Q, \cdot)$ is not a group.

Theorem 2.7. $4(N Q,+, \cdot)$ is a commutative ring.

Theorem 2.8. ${ }^{34}(N Q,+)=\left\{(a, b T, c I, d F) \mid a, b, c, d \in \mathbb{R}\right.$ or $\mathbb{C}$ or $\mathbb{Z}_{p} ; p$ a prime, +$\}$ be the Neutrosophic quadruple group. Then $V=(N Q,+, o)$ is a Neutrosophic Quadruple vector space $(N Q-$ vectorspace) over $\mathbb{R}$ or $\mathbb{C}$ or $\mathbb{Z}_{p}$, where' $o^{\prime}$ is the special type of operation between $V$ and $\mathbb{R}$ (or $\mathbb{C}$ or $\mathbb{Z}_{p}$ ) defined as scalar multiplication.

Definition 2.9. ${ }^{34}$ Let $V=(N Q,+)$ be a $N Q$ vector space over $\mathbb{R}$ (or $\mathbb{C}$ or $\mathbb{Z}_{p}$ ). A subset $L$ of $V$ is said to be $N Q$ linearly dependent or simply dependent, if there exists distinct vectors $a_{1}, a_{2}, \cdots, a_{k} \in L$ and scalars $d_{1}, d_{2}, \cdots, d_{k} \in \mathbb{R}\left(\right.$ or $\left.\mathbb{C} \circ \mathbb{Z}_{p}\right)$ not all zero such that $d_{1} \circ a_{1}+d_{2} \circ a_{2}+\cdots+d_{k} \circ a_{k}=0$.

We say the set of vectors $a_{1}, a_{2}, \cdots, a_{k}$ is $N Q$ linearly independent if it is not $N Q$ linearly dependent. 
Definition 2.10. ${ }^{34}$ Let $V=\left(N Q,+\right.$ ) be a $N Q$ vector space over $\mathbb{R}$ (or $\mathbb{C}$ or $\mathbb{Z}_{p}$ ). A subset $W$ of $V$ is said to be Neutrosophic Quadruple vector subspace of $V$ if $W$ itself is a Neutrosophic Quadruple vector space over $\mathbb{R}$ (or $\mathbb{C}$ or $\mathbb{Z}_{p}$ ).

Definition 2.11. ${ }^{1}$ Let $P(V)$ be the power set of a set $V, P^{*}(V)=P(V)-\{\emptyset\}$ and let $\mathrm{K}$ be a field.

The quadruple $(V,+, \bullet, K)$ is called a hypervector space over a field $\mathrm{K}$ if:

1. $(V,+)$ is an abelian group.

2. $\bullet: K \times V \longrightarrow P^{*}(V)$ is a hyperoperation such that for all $k, m \in K$ and $u, v \in V$, the following conditions hold:
(a) $(k+m) \bullet u \subseteq(k \bullet u)+(m \bullet u)$,
(b) $k \bullet(u+v) \subseteq(k \bullet u)+(k \bullet v)$,
(c) $k \bullet(m \bullet u)=(k m) \bullet u$, where $k \bullet(m \bullet u)=\{k \bullet v: v \in m \bullet u\}$,
(d) $(-k) \bullet u=k \bullet(-u)$,
(e) $u \in 1 \bullet u$.

A hypervector space is said to be strongly left distributive (resp. strongly right distributive) if equality holds in (a) (resp. in (b)). $(V,+, \bullet, K)$ is called a strongly distributive hypervector space if it is both strongly left and strongly right distributive.

Definition 2.12. ${ }^{1}$ Let $(V,+, \bullet, K)$ be any strongly distributive hypervector space over a field $\mathrm{K}$ and let

$$
V(I)=<V \cup(I)>=\{u=(a, b I): a, b \in V\}
$$

be a set generated by $V$, and $I$. The quadruple $(V(I),+, \bullet, K)$ is called a weak neutrosophic strongly distributive hyper vector space over a field $\mathrm{K}$.

For every element $u=(a, b I), v=(d, e I) \in V(I)$, and $k \in K$ we define

$$
\begin{gathered}
u+v=(a+d,(b+e) I \in V(I), \\
k \bullet u=\{(x, y I): x \in k \bullet a, y \in k \bullet b\} .
\end{gathered}
$$

If $\mathrm{K}$ is a neutrosophic field, that is, $K=K(I)$, then the quadruple $(V(I),+, \bullet, K(I))$ is called a strong neutrosophic strongly distributive hyper vector space over a neutrosophic field $K(I)$.

For every element $u=(a, b I), v=(d, e I) \in V(I)$, and $\alpha=(k, m I) \in K(I)$, we define

$$
\begin{gathered}
u+v=(a, b I)+(d, e I)=(a+d,(b+e) I), \\
\alpha \bullet u=\{(x, y I):(x \in k \bullet a, y \in k \bullet b \cup m \bullet a \cup m \bullet b)\} .
\end{gathered}
$$

The elements of $V(I)$ are called neutrosophic vectors and the elements of $K(I)$ are called neutrosophic scalars. The zero neutrosophic vector of $V(I),(0,0 I)$, is denoted by $\theta$, the zero element $0 \in K$ is represented by $(0,0 I)$ in $K(I)$ and $1 \in K$ is represented by $(1,0 I) \in K(I)$.

Theorem 2.13. ${ }^{\square}$ Every strong neutrosophic hypervector space is a weak neutrosophic hy-pervector space

Theorem 2.14. I Every weak neutrosophic hypervector space is a strongly distributive hypervector space

\section{Formulation of a Neutrosophic Quadruple(NQ) Hypervector Spaces and its Subspaces}

In this section, we develop the concept of neutrosophic quadruple hypervector spaces and present some of their basic properties. Except otherwise stated, all neutrosophic quadruple numbers will be real neutrosophic quadruple numbers of the form $(a, b T, c I, d F)$ where $a, b, c, d \in \mathbb{R}$. The elements of $V(T, I, F)$ will be called neutrosophic quadruple vectors and the elements of $K(I)$ and $K(T, I, F)$ will be called neutrosophic scalars and neutrosophic quadruple scalars respectively. $(0,0 T, 0 I, 0 F)$, the zero vector of $V(T, I, F)$ will be denoted by $\theta$, the zero element of $K(T, I, F)$ will be denoted by $0 \in K$ while $1 \in K$ will be denoted by $(1,0 T, 0 I, 0 F)$ in $K(T, I, F)$. 
Definition 3.1. Let $(V,+, \bullet, K)$ be any strongly distributive hypervector space over a field $\mathrm{K}$ and let

$$
V(T, I, F)=<V \cup(T, I, F)>=\{u=(a, b T, c I, d F): a, b, c, d \in V\} .
$$

be a set generated by $V, T, I$ and $F$. The quadruple $(V(T, I, F),+, \bullet, K)$ is called a weak neutrosophic quadruple strongly distributive hypervector space over a field $\mathrm{K}$.

For every element $u=(a, b T, c I, d F), v=(e, f T, g I, h F) \in V(T, I, F)$ and $k \in K$ we define

$$
\begin{gathered}
u+v=(a+e,(b+f) T,(c+g) I,(d+h) F) \in V(T, I, F), \\
k \bullet u=\{(r, x T, y I, z F): r \in k \bullet a, x \in k \bullet b, y \in k \bullet c, z \in k \bullet d\} .
\end{gathered}
$$

Definition 3.2. Let $(V,+, \bullet, K)$ be any strongly distributive hypervector space over a field $\mathrm{K}$ and let

$$
V(T, I, F)=<V \cup(T, I, F)>=\{u=(a, b T, c I, d F): a, b, c, d \in V\} .
$$

be a set generated by $V, T, I$ and $F$. The quadruple $(V(T, I, F),+, \bullet, K(I))$ is called a strong neutrosophic quadruple strongly distributive hypervector space over a neutrosophic field $K(I)$. define

For every element $u=(a, b T, c I, d F), v=(e, f T, g I, h F) \in V(T, I, F)$ and $\alpha=(k, m I) \in K(I)$, we

$$
\begin{gathered}
u+v=(a+e,(b+f) T,(c+g) I,(d+h) F) \in V(T, I, F), \\
\alpha \bullet u=\{(r, x T, y I, z F):(r \in k \bullet a, x \in k \bullet b, y \in k \bullet c \cup m \bullet a \cup m \bullet b \cup m \bullet c, z \in k \bullet d \cup m \bullet d)\} .
\end{gathered}
$$

Definition 3.3. Let $(V,+, \bullet, K)$ be any strongly distributive hypervector space over a field $\mathrm{K}$ and let

$$
V(T, I, F)=<V \cup(T, I, F)>=\{u=(a, b T, c I, d F): a, b, c, d \in V\}
$$

be a set generated by $V, T, I$ and $F$.

The quadruple $(V(T, I, F),+, \bullet, K(T, I, F))$ is called a super strong neutrosophic quadruple strongly distributive hypervector space over a neutrosophic field $K(T, I, F)$.

For every element $u=(a, b T, c I, d F), v=(e, f T, g I, h F) \in V(T, I, F)$ and $\alpha=(k, m T, n I, t F) \in$ $K(T, I, F)$, we define

$$
u+v=(a+e,(b+f) T,(c+g) I,(d+h) F) \in V(T, I, F),
$$

$\alpha \bullet u=\{(r, x T, y I, z F): r \in k \bullet a, x \in k \bullet b \cup m \bullet a \cup m \bullet b, y \in k \bullet c \cup m \bullet c \cup n \bullet a \cup n \bullet b \cup n \bullet c$,

$z \in k \bullet d \cup m \bullet d \cup n \bullet d \cup t \bullet a \cup t \bullet b \cup t \bullet c \cup t \bullet d\}$.

Example 3.4. Let $\mathrm{n}$ be a positive integer and let $V(T, I, F)=\mathbb{R}^{n}(T, I, F)$ denote the neutrosophic quadruple set of column neutrosophic quadruple vectors of length $n$ with entries from the field $\mathbb{R}$ :

$$
\mathbb{R}^{n}(T, I, F)=\left\{\left(\begin{array}{c}
\left(a_{1}, b_{1} T, c_{1} I, d_{1} F\right) \\
\left(a_{2}, b_{2} T, c_{2} I, d_{2} F\right) \\
\vdots \\
\left(a_{n}, b_{n} T, c_{n} I, d_{n} F\right)
\end{array}\right): a_{i}, b_{i}, c_{i}, d_{i} \in \mathbb{R}, \quad i=1,2 \cdots n\right\}
$$

For all

$$
u=\left(\begin{array}{c}
\left(a_{1}, b_{1} T, c_{1} I, d_{1} F\right) \\
\left(a_{2}, b_{2} T, c_{2} I, d_{2} F\right) \\
\vdots \\
\left(a_{n}, b_{n} T, c_{n} I, d_{n} F\right)
\end{array}\right), v=\left(\begin{array}{c}
\left(e_{1}, f_{1} T, g_{1} I, h_{1} F\right) \\
\left(e_{2}, f_{2} T, g_{2} I, h_{2} F\right) \\
\vdots \\
\left(e_{n}, f_{n} T, g_{n} I, h_{n} F\right)
\end{array}\right) \in V(T, I, F)
$$

and $k \in K$ define:

$$
\begin{aligned}
u+v= & \left(\begin{array}{c}
\left(a_{1}, b_{1} T, c_{1} I, d_{1} F\right) \\
\left(a_{2}, b_{2} T, c_{2} I, d_{2} F\right) \\
\vdots \\
\left(a_{n}, b_{n} T, c_{n} I, d_{n} F\right)
\end{array}\right)+\left(\begin{array}{c}
\left(e_{1}, f_{1} T, g_{1} I, h_{1} F\right) \\
\left(e_{2}, f_{2} T, g_{2} I, h_{2} F\right) \\
\vdots \\
\left(e_{n}, f_{n} T, g_{n} I, h_{n} F\right)
\end{array}\right) \\
= & \left(\begin{array}{c}
\left(a_{1}+e_{1},\left(b_{1}+f_{1}\right) T,\left(c_{1}+g_{1}\right) I,\left(d_{1}+h_{1}\right) F\right) \\
\left(a_{2}+e_{2},\left(b_{2}+f_{2}\right) T,\left(c_{2}+g_{2}\right) I,\left(d_{2}+h_{2}\right) F\right) \\
\vdots \\
\left(a_{n}+e_{n},\left(b_{n}+f_{n}\right) T,\left(c_{n}+g_{n}\right) I,\left(d_{n}+h_{n}\right) F\right)
\end{array}\right)
\end{aligned}
$$

Doi :10.5281/zenodo.3752906 
and

$k \bullet\left(\begin{array}{c}\left(a_{1}, b_{1} T, c_{1} I, d_{1} F\right) \\ \left(a_{2}, b_{2} T, c_{2} I, d_{2} F\right) \\ \vdots \\ \left(a_{n}, b_{n} T, c_{n} I, d_{n} F\right)\end{array}\right)=\left\{\left(\begin{array}{c}\left(r_{1}, x_{1} T, y_{1} I, z_{1} F\right) \\ \left(r_{2}, x_{2} T, y_{2} I, z_{2} F\right) \\ \vdots \\ \left(r_{n}, x_{n} T, y_{n} I, z_{n} F\right)\end{array}\right) \begin{array}{c}r_{1} \in k \bullet a_{1}, x_{1} \in k \bullet b_{1}, y_{1} \in k \bullet c_{1}, z_{1} \in k \bullet d_{1} \\ r_{2} \in k \bullet a_{2}, x_{2} \in k \bullet b_{2}, y_{2} \in k \bullet c_{2}, z_{2} \in k \bullet d_{2} \\ \vdots \\ r_{n} \in k \bullet a_{n}, x_{n} \in k \bullet b_{n}, y_{n} \in k \bullet c_{n}, z_{n} \in k \bullet d_{n}\end{array}\right\}$.

Then $(V(T, I, F),+, \bullet, K)$ is a weak neutrosophic quadruple strongly distributive hypervector space over the field $\mathrm{K}$.

Example 3.5. Let $V(T, I, F)=R^{2}(T, I, F)$ and let $K=R(I)$. For all

$u=\left(\left(a_{1}, b_{1} T, c_{1} I, d_{1} F\right),\left(e_{1}, f_{1} T, g_{1} I, h_{1} F\right)\right), v=\left(\left(a_{2}, b_{2} T, c_{2} I, d_{2} F\right),\left(e_{2}, f_{2} T, g_{2} I, h_{2} F\right)\right) \in V(T, I, F)$ and $\alpha=(k, m I) \in K(I)$, define:

$$
\begin{aligned}
u+v=\left(\left(a_{1}+\right.\right. & \left.\left.a_{2},\left(b_{1}+b_{2}\right) T,\left(c_{1}+c_{2}\right) I,\left(d_{1}+d_{2}\right) F\right),\left(e_{1}+e_{2},\left(f_{1}+f_{2}\right) T,\left(g_{1}+g_{2}\right) I,\left(h_{1}+h_{2}\right) F\right)\right) . \\
\alpha \bullet u= & \{((r, x T, y I, z F),(p, q T, s I, t F)): \\
& \left(r \in k \bullet a_{1}, x \in k \bullet b_{1}, y \in k \bullet c_{1} \cup m \bullet a_{1} \cup m \bullet b_{1} \cup m \bullet c_{1}, z \in k \bullet d_{1} \cup m \bullet d_{1}\right) \\
& \left.\left(p \in k \bullet e_{1}, q \in k \bullet f_{1}, s \in k \bullet g_{1} \cup m \bullet e_{1} \cup m \bullet f_{1} \cup m \bullet g_{1}, t \in k \bullet h_{1} \cup m \bullet h_{1}\right)\right\} .
\end{aligned}
$$

Then $(V(T, I, F),+, \bullet, K(I))$ is an strong neutrosophic quadruple strongly distributive hypervector space over the neutrosophic field $K(I)$.

Example 3.6. Let $V(T, I, F)=R^{2}(T, I, F)$ and let $K=R(T, I, F)$. For all $u=\left(\left(a_{1}, b_{1} T, c_{1} I, d_{1} F\right),\left(e_{1}, f_{1} T, g_{1} I, h_{1} F\right)\right), v=\left(\left(a_{2}, b_{2} T, c_{2} I, d_{2} F\right),\left(e_{2}, f_{2} T, g_{2} I, h_{2} F\right)\right) \in V(T, I, F)$ and $\alpha=(k, m T, n I, w F) \in K(T, I, F)$, define:

$$
\begin{aligned}
u+v=( & \left.\left(a_{1}+a_{2},\left(b_{1}+b_{2}\right) T,\left(c_{1}+c_{2}\right) I,\left(d_{1}+d_{2}\right) F\right),\left(e_{1}+e_{2},\left(f_{1}+f_{2}\right) T,\left(g_{1}+g_{2}\right) I,\left(h_{1}+h_{2}\right) F\right)\right) . \\
\alpha \bullet u= & \{((r, x T, y I, z F),(p, q T, s I, t F)): \\
& \left(r \in k \bullet a_{1}, x \in k \bullet b_{1} \cup m \bullet a_{1} \cup m \bullet b_{1}, y \in k \bullet c_{1} \cup m \bullet c_{1} \cup n \bullet a_{1} \cup n \bullet b_{1} \cup n \bullet c_{1},\right. \\
& \left.z \in k \bullet d_{1} \cup m \bullet d_{1} \cup n \bullet d_{1} \cup w \bullet a_{1} \cup w \bullet b_{1} \cup w \bullet c_{1} \cup w \bullet d_{1}\right) \\
& \left(p \in k \bullet e_{1}, q \in k \bullet f_{1} \cup m \bullet e_{1} \cup m \bullet f_{1}, s \in k \bullet g_{1} \cup m \bullet g_{1} \cup n \bullet e_{1} \cup n \bullet f_{1} \cup n \bullet g_{1},\right. \\
& \left.\left.t \in k \bullet h_{1} \cup m \bullet h_{1} \cup n \bullet h_{1} \cup w \bullet e_{1} \cup w \bullet f_{1} \cup w \bullet g_{1} \cup w \bullet h_{1}\right)\right\} .
\end{aligned}
$$

Then $(V(T, I, F),+, \bullet, K(T, I, F))$ is a super strong neutrosophic quadruple strongly distributive hypervector space over the neutrosophic quadruple field $K(T, I, F)$.

From here on, every weak( strong [super strong]) neutrosophic quadruple strongly distributive hypervector space will simply be called a weak( resp.(strong [super strong])) NQ-Hypervector space.

\section{Proposition 3.7. .}

1. Every super strong NQ-Hypervector space is a strong NQ-Hypervector space.

2. Every super strong NQ-Hypervector space is a weak NQ-Hypervector space.

3. Every strong NQ-Hypervector space is a weak NQ-Hypervector space.

Proof:

1. This is true, since $K(I) \subseteq K(T, I, F)$.

2. This is true, since $K \subseteq K(T, I, F)$.

3. This is true, since $K \subseteq K(I)$.

Proposition 3.8. Every weak NQ-Hypervector space is a strongly distributive hypervector space.

Proof: Suppose that $V(T, I, F)$ is a weak $N Q$-Hypervector space over a field $K$.

That $(N Q,+)$ is a vector space is seen in [4] $]$.

Let $u=(a, b T, c I, d F), v=(e, f T, g I, h F) \in V(T, I, F)$ and $k, m \in K$ be arbitrary. Then 


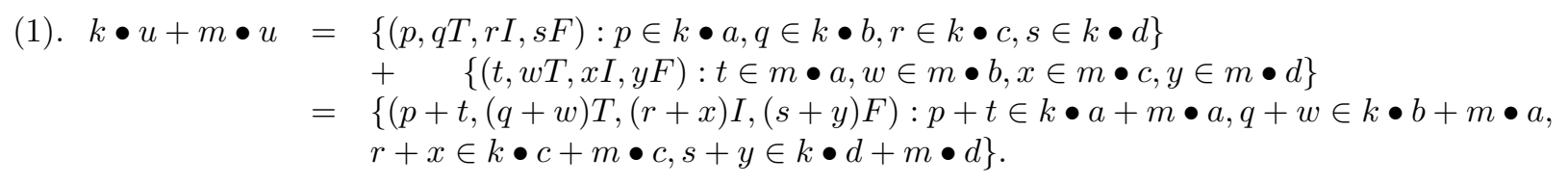

Also

$$
\begin{aligned}
& =(k m) \bullet(a, b T, c I, d F) \\
& =(k m) \bullet u \text {. } \\
& =k \bullet(-u) \text {. }
\end{aligned}
$$

Showing that $u \in 1 \bullet u$.

Therefore we say that $V(T, I, F)$ is a strongly distributive hypervector space.

Proposition 3.9. Let $V(T, I, F)$ be a super strong(strong) NQ-Hypervector space over a neutrosophic quadruple field $K(T, I, F)$ (neutrosophic field $K(I)$ ). Then

1. $V(T, I, F)$ generally is not a strongly distributive hypervector space.

2. $V(T, I, F)$ always contain a strongly distributive hypervector space

Proposition 3.10. Let $\left(V(T, I, F),+_{1}, \bullet_{1}\right)$ and $\left(U(T, I, F),+_{2}, \bullet_{2}\right)$ be any two super strong NQ-Hypervector space over a neutrosophic quadruple field $K(T, I, F)$. Let

$$
\begin{aligned}
V(T, I, F) \times U(T, I, F)= & \left\{\left(\left(v, v_{1} T, v_{2} I, v_{3} F\right),\left(u, u_{1} T, u_{2} I, u_{3} F\right)\right):\right. \\
& \left.\left(v, v_{1} T, v_{2} I, v_{3} F\right) \in V(T, I, F),\left(u, u_{1} T, u_{2} I, u_{3} F\right) \in U(T, I, F)\right\}
\end{aligned} .
$$

For all

$x=\left(\left(v, v_{1} T, v_{2} I, v_{3} F\right),\left(u, u_{1} T, u_{2} I, u_{3} F\right)\right), y=\left(\left(v^{\prime}, v_{1}^{\prime} T, v_{2}^{\prime} I, v_{3}^{\prime} F\right),\left(u^{\prime}, u_{1}^{\prime} T, u_{2}^{\prime} I, u_{3}^{\prime} F\right)\right) \in V(T, I, F) \times U(T, I, F)$

and $\alpha=\left(k, k_{1} T, k_{2} I, k_{3} F\right) \in K(T, I, F)$

$$
\begin{aligned}
x+y=( & \left.\left(v+v^{\prime},\left(v_{1}+v_{1}^{\prime}\right) T,\left(v_{2}+v_{2}^{\prime}\right) I,\left(v_{3}+v_{3}^{\prime}\right) F\right),\left(u+u^{\prime},\left(u_{1}+u_{1}^{\prime}\right) T,\left(u_{2}+u_{2}^{\prime}\right) I,\left(u_{3}+u_{3}^{\prime}\right) F\right)\right) . \\
\alpha \bullet x= & \left\{\left(\left(p, p_{1} T, p_{2} I, p_{3} F\right),\left(q, q_{1} T, q_{2} I, q_{3} F\right)\right):\right. \\
& \left(p \in k \bullet v, p_{1} \in k \bullet v_{1} \cup k_{1} \bullet v \cup k_{1} \bullet v_{1}, p_{2} \in k \bullet v_{2} \cup k_{1} \bullet v_{2} \cup k_{2} \bullet v \cup k_{2} \bullet v_{1} \cup k_{2} \bullet v_{2},\right. \\
& \left.p_{3} \in k \bullet v_{3} \cup k_{1} \bullet v_{3} \cup k_{2} \bullet v_{3} \cup k_{3} \bullet v \cup k_{3} \bullet v_{1} \cup k_{3} \bullet v_{2} \cup k_{3} \bullet v_{3}\right) \\
& \left(q \in k \bullet u, q_{1} \in k \bullet u_{1} \cup k_{1} \bullet u \cup k_{1} \bullet u_{1}, q_{2} \in k \bullet u_{2} \cup k_{1} \bullet u_{2} \cup k_{2} \bullet u \cup k_{2} \bullet u_{1} \cup k_{2} \bullet u_{2},\right. \\
& \left.\left.q_{3} \in k \bullet u_{3} \cup k_{1} \bullet u_{3} \cup k_{2} \bullet u_{3} \cup k_{3} \bullet u \cup k_{3} \bullet u_{1} \cup k_{3} \bullet u_{2} \cup k_{3} \bullet u_{3}\right)\right\} .
\end{aligned}
$$

Then $(V(T, I, F) \times U(T, I, F),+, \bullet, K(T, I, F))$ is a super strong NQ-Hypervector space. 
Proposition 3.11. Let $\left(V(T, I, F),+_{1}, \bullet_{1}\right)$ and $\left(U(T, I, F),+_{2}, \bullet_{2}\right)$ be any two strong NQ-Hypervector space over a neutrosophic field $K(I)$. Let

$$
\begin{aligned}
V(T, I, F) \times U(T, I, F)= & \left\{\left(\left(v, v_{1} T, v_{2} I, v_{3} F\right),\left(u, u_{1} T, u_{2} I, u_{3} F\right)\right):\right. \\
& \left.\left(v, v_{1} T, v_{2} I, v_{3} F\right) \in V(T, I, F),\left(u, u_{1} T, u_{2} I, u_{3} F\right) \in U(T, I, F)\right\}
\end{aligned}
$$

for all

$x=\left(\left(v, v_{1} T, v_{2} I, v_{3} F\right),\left(u, u_{1} T, u_{2} I, u_{3} F\right)\right), y=\left(\left(v^{\prime}, v_{1}^{\prime} T, v_{2}^{\prime} I, v_{3}^{\prime} F\right),\left(u^{\prime}, u_{1}^{\prime} T, u_{2}^{\prime} I, u_{3}^{\prime} F\right)\right) \in V(T, I, F) \times$ $U(T, I, F)$ and $\alpha=\left(k, k_{1} I\right) \in K(I)$

$$
\begin{aligned}
x+y=( & \left.\left(v+v^{\prime},\left(v_{1}+v_{1}^{\prime}\right) T,\left(v_{2}+v_{2}^{\prime}\right) I,\left(v_{3}+v_{3}^{\prime}\right) F\right),\left(u+u^{\prime},\left(u_{1}+u_{1}^{\prime}\right) T,\left(u_{2}+u_{2}^{\prime}\right) I,\left(u_{3}+u_{3}^{\prime}\right) F\right)\right) . \\
\alpha \bullet x= & \left\{\left(\left(p, p_{1} T, p_{2} I, p_{3} F\right),\left(q, q_{1} T, q_{2} I, q_{3} F\right)\right):\right. \\
& \left(p \in k \bullet v, p_{1} \in k \bullet v_{1}, p_{2} \in k \bullet v_{2} \cup k_{1} \bullet v \cup k_{1} \bullet v_{1} \cup k_{1} \bullet v_{2}, p_{3} \in k \bullet v_{3} \cup k_{1} \bullet v_{3}\right) \\
& \left.\left(q \in k \bullet u, q_{1} \in k \bullet u_{1}, q_{2} \in k \bullet u_{2} \cup k_{1} \bullet u \cup k_{1} \bullet u_{1} \cup k_{1} \bullet u_{2}, q_{3} \in k \bullet u_{3} \cup k_{1} \bullet u_{3}\right)\right\} .
\end{aligned}
$$

Then $(V(T, I, F) \times U(T, I, F),+, \bullet, K(I))$ is a strong NQ-Hypervector space.

Proposition 3.12. Let $\left(V(T, I, F),+_{1}, \bullet_{1}\right)$ and $\left(U(T, I, F),+_{2}, \bullet_{2}\right)$ be any two weak $N Q$-Hypervector spaces over a field K. Let

$$
\begin{aligned}
V(T, I, F) \times U(T, I, F)= & \left\{\left(\left(v, v_{1} T, v_{2} I, v_{3} F\right),\left(u, u_{1} T, u_{2} I, u_{3} F\right)\right):\right. \\
& \left.\left(v, v_{1} T, v_{2} I, v_{3} F\right) \in V(T, I, F),\left(u, u_{1} T, u_{2} I, u_{3} F\right) \in U(T, I, F)\right\} .
\end{aligned}
$$

For all

$x=\left(\left(v, v_{1} T, v_{2} I, v_{3} F\right),\left(u, u_{1} T, u_{2} I, u_{3} F\right)\right), y=\left(\left(v^{\prime}, v_{1}^{\prime} T, v_{2}^{\prime} I, v_{3}^{\prime} F\right),\left(u^{\prime}, u_{1}^{\prime} T, u_{2}^{\prime} I, u_{3}^{\prime} F\right)\right) \in V(T, I, F) \times$ $U(T, I, F)$ and $k \in K$

$$
\begin{aligned}
x+y= & \left(\left(v+v^{\prime},\left(v_{1}+v_{1}^{\prime}\right) T,\left(v_{2}+v_{2}^{\prime}\right) I,\left(v_{3}+v_{3}^{\prime}\right) F\right),\left(u+u^{\prime},\left(u_{1}+u_{1}^{\prime}\right) T,\left(u_{2}+u_{2}^{\prime}\right) I,\left(u_{3}+u_{3}^{\prime}\right) F\right)\right) . \\
k \bullet x= & \left\{\left(\left(p, p_{1} T, p_{2} I, p_{3} F\right),\left(q, q_{1} T, q_{2} I, q_{3} F\right)\right):\left(p \in k \bullet v, p_{1} \in k \bullet v_{1}, p_{2} \in k \bullet v_{2}, p_{3} \in k \bullet v_{3}\right)\right. \\
& \left.\left(q \in k \bullet u, q_{1} \in k \bullet u_{1}, q_{2} \in k \bullet u_{2}, q_{3} \in k \bullet u_{3}\right)\right\} .
\end{aligned}
$$

Then $(V(T, I, F) \times U(T, I, F),+, \bullet, K)$ is a weak NQ-Hypervector space.

Proposition 3.13. Let $V(T, I, F)$ be any super strong $N Q$-Hypervector space over a neutrosophic quadruple field $K(T, I, F)$, let $U(T, I, F)$ be any strong $N Q$-Hypervector space over a neutrosophic field $K(I)$ and let $W(T, I, F)$ be any weak $N Q$-Hypervector space over a field $K$. Then

1. $(V(T, I, F) \times U(T, I, F),+, \bullet, K(I))$ is a strong NQ-Hypervector space.

2. $(V(T, I, F) \times W(T, I, F),+, \bullet, K)$ is a weak $N Q$-Hypervector space.

3. $(U(T, I, F) \times W(T, I, F),+, \bullet, K)$ is a weak NQ-Hypervector space.

Proof:

1. From 1 of 3.7, we know that every super strong NQ-Hypervector space is a strong NQ-Hypervector space. Then by applying 3.11 to this, we obtained the required result.

2. From 2 of 3.7. we know that every super strong NQ-Hypervector space is a weak NQ-Hypervector space. Then by 3.12 the proof follows .

3. From 3 of 3.7, we know that every strong NQ-Hypervector space is a weak NQ-Hypervector space. Then by 3.12 the proof follows .

Definition 3.14. A nonempty subset $N(T, I, F)$ of a super strong NQ-Hypervector space

$(V(T, I, F),+, \bullet, K(T, I, F))$ over a neutrosophic quadruple field $K(T, I, F)$ is called a super strong NQHypersubspace of $V(T, I, F)$ if $(N(T, I, F),+, \bullet, K(T, I, F))$ is itself a super strong NQ-Hypervector space over $K(T, I, F)$. It is essential that $N(T, I, F)$ contains a proper subset which is a Hypervector space over $\mathrm{K}$.

Definition 3.15. A nonempty subset $N(T, I, F)$ of a strong NQ-Hypervector space

$(V(T, I, F),+, \bullet, K(I))$ over a neutrosophic field $K(I)$ is called a strong NQ-Hypersubspace of $V(T, I, F)$ if $(N(T, I, F),+, \bullet, K(I))$ is itself a strong NQ-Hypervector space over $K(I)$. It is essential that $N(T, I, F)$ contains a proper subset which is a Hypervector space over $\mathrm{K}$. 
Proposition 3.16. Let $N[T, I, F]$ be a subset of a super strong NQ-Hypervector space $(V(T, I, F),+, \bullet, K(T, I, F))$ over a neutrosophic quadruple field $K(T, I, F)$. Then $N(I, T, F)$ is a super strong NQ-Hypersubspace of $V(T, I, F)$ if and only if for all $u=(a, b T, c I, d F), v=(e, f T, g I, h F) \in$ $V(T, I, F)$ and $\alpha=(k, m T, n I, t F) \in K(T, I, F)$ the following conditions hold:

1. $N[T, I, F] \neq \emptyset$,

2. $u+v \in N[T, I, F]$,

3. $\alpha \bullet v \subseteq N[T, I, F]$,

4. $N[T, I, F]$ contains a proper subset which is a hypervector space over $K$.

Proof:

If $N(T, I, F)$ is a super strong NQ-Hypersubspace of $V(T, I, F)$, then obviously conditions $1,2,3$ and 4 hold.

Conversely, let $N[T, I, F]$ be a subset of $V(T, I, F)$ such that $N(T, I, F)$ satisfies the four conditions $1,2,3$ and 4.

To proof that $N(T, I, F)$ is a NQ-Hypersubspace of $V(T, I, F)$. It is enough to prove that

1. $N(T, I, F)$ has a zero NQ-vector.

2. Each NQ-vector in $N(T, I, F)$ has an additive inverse.

Since $N(T, I, F)$ is non-empty, let $u=(a, b T, c I, d F) \in N(T, I, F)$.

Now for $(0,0 T, 0 I, 0 F) \in K(T, I, F)$ and by condition 3 we have that

$$
(0,0 T, 0 I, 0 F) \bullet u=(0,0 T, 0 I, 0 F) \bullet(a, b T, c I, d F) \subseteq N(T, I, F) \Longrightarrow \theta \in N(I, T, F) .
$$

Therefore $N(T, I, F)$ has a zero vector. Again, since $-(1,0 T, 0 I, 0 F) \in K(T, I, F)$ then $-(1,0 T, 0 I, 0 F) \bullet u=-(1,0 T, 0 I, 0 F) \bullet(a, b T, c I, d F) \subseteq N \Longrightarrow-u \in N(T, I, F)$.

Hence each NQ-vector in $N(T, I, F)$ has an additive inverse.

Proposition 3.17. Let $N[T, I, F]$ be a subset of a strong NQ-Hypervector space

$(V(T, I, F),+, \bullet, K(I))$ over a neutrosophic field $K(I)$. Then $N(I, T, F)$ is a strong NQ-hypersubspace of $V(T, I, F)$ if and only if for all $u=(a, b T, c I, d F), v=(e, f T, g I, h F) \in V(T, I, F)$ and $\alpha=(k, m I) \in$ $K(I)$ the following conditions hold:

1. $N[T, I, F] \neq \emptyset$,

2. $u+v \in N[T, I, F]$

3. $\alpha \bullet v \subseteq N[T, I, F]$

4. $N[T, I, F]$ contains a proper subset which is a hypervector space over $K$

Proof : Follow similar approach as the proof of 3.16 , above.

Corollary 3.18. Let $N[T, I, F]$ be a NQ-hypersubspace of a $N Q$-hypervector space $V(T, I, F)$ if and only if

1. $N[T, I, F]$ is non-empty.

2. $\alpha \bullet u+\beta \bullet v \subseteq N[T, I, F]$, for all $\alpha=\left(k_{1}, m_{1} T, n_{1} I, r_{1} F\right), \beta=\left(k_{2}, m_{2} T, n_{2} I, r_{2} F\right) \in K(T, I, F)$ and $u=(a, b T, c I, d F), v=(e, f T, g I, h F) \in N[T, I, F]$.

3. $N[T, I, F]$ contains a proper subset which is a hypervector space over $K$.

Example 3.19. Let $V(T, I, F)$ be a super strong NQ-Hypervector space defined in Example 3.6

Let $N(T, I, F)=K(T, I, F) \times\{(0,0 T, 0 I, 0 F)\} \subseteq V(T, I, F)$

Then $N(T, I, F)$ is a super strong NQ-Hypersubspace

Proof: Since $\theta=((0,0 T, 0 I, 0 F),(0,0 T, 0 I, 0 F)) \in N(T, I, F)$. Then $N(T, I, F) \neq \emptyset$ Now let

$u=\left(\left(a_{1}, b_{1} T, c_{1} I, d_{1} F\right),(0,0 T, 0 I, 0 F)\right), v=\left(\left(a_{2}, b_{2} T, c_{2} I, d_{2} F\right),(0,0 T, 0 I, 0 F)\right) \in N(T, I, F)$ and

$\alpha=(k, m T, n I, w F), \beta=\left(k^{\prime}, m^{\prime} T, n^{\prime} I, w^{\prime} F\right) \in K(T, I, F)$, with $a_{1}, b_{1}, c_{1}, d_{1}, a_{2}, b_{2}, c_{2}, d_{2} \in N$ and

$k, m, n, w, k^{\prime}, m^{\prime}, n^{\prime}, w^{\prime} \in K$ 
Then $\alpha \bullet u+\beta \bullet v$

$=(k, m T, n I, w F) \bullet\left[\left(a_{1}, b_{1} T, c_{1} I, d_{1} F\right),(0,0 T, 0 I, 0 F)\right]+\left(k^{\prime}, m^{\prime} T, n^{\prime} I, w^{\prime} F\right) \bullet\left[\left(a_{2}, b_{2} T, c_{2} I, d_{2} F\right),(0,0 T, 0 I, 0 F)\right]$

$\subseteq\left\{((x, y T, z I, t F),(p, q T, r I, s F)): x \in k \bullet a_{1}, y \in k \bullet b_{1} \cup m \bullet a_{1} \cup m \bullet b_{1}, z \in k \bullet c_{1} \cup m \bullet\right.$

$c_{1} \cup n \bullet a_{1} \cup n \bullet b_{1} \cup n \bullet c_{1}, t \in k \bullet d_{1} \cup m \bullet d_{1} \cup n \bullet d_{1} \cup w \bullet a_{1} \cup w \bullet b_{1} \cup w \bullet c_{1} \cup w \bullet d_{1}, p \in k \bullet 0, q \in$ $k \bullet 0 \cup m \bullet 0 \cup m \bullet 0, r \in k \bullet 0 \cup m \bullet 0 \cup n \bullet 0 \cup n \bullet 0 \cup n \bullet 0, s \in k \bullet 0 \cup m \bullet 0 \cup n \bullet 0 \cup w \bullet 0 \cup w \bullet 0 \cup w \bullet 0 \cup w \bullet 0\}$ $+\left\{\left(\left(x^{\prime}, y^{\prime} T, z^{\prime} I, t^{\prime} F\right),\left(p^{\prime}, q^{\prime} T, r^{\prime} I, s^{\prime} F\right)\right): x^{\prime} \in k^{\prime} \bullet a_{2}, y^{\prime} \in k^{\prime} \bullet b_{2} \cup m^{\prime} \bullet a_{2} \cup m^{\prime} \bullet b_{2}, z^{\prime} \in k^{\prime} \bullet c_{2} \cup m^{\prime} \bullet\right.$ $c_{2} \cup n^{\prime} \bullet a_{2} \cup n^{\prime} \bullet b_{2} \cup n^{\prime} \bullet c_{2}, t^{\prime} \in k^{\prime} \bullet d_{2} \cup m^{\prime} \bullet d_{2} \cup n^{\prime} \bullet d_{2} \cup w^{\prime} \bullet a_{2} \cup w^{\prime} \bullet b_{2} \cup w^{\prime} \bullet c_{2} \cup w^{\prime} \bullet d_{2}, p^{\prime} \in k^{\prime} \bullet 0, q^{\prime} \in$ $\left.k^{\prime} \bullet 0 \cup m^{\prime} \bullet 0 \cup m^{\prime} \bullet 0, r^{\prime} \in k^{\prime} \bullet 0 \cup m^{\prime} \bullet 0 \cup n^{\prime} \bullet 0 \cup n^{\prime} \bullet 0 \cup n^{\prime} \bullet 0, s^{\prime} \in k \bullet 0 \cup m^{\prime} \bullet 0 \cup n^{\prime} \bullet 0 \cup w^{\prime} \bullet 0 \cup w^{\prime} \bullet 0 \cup w^{\prime} \bullet 0 \cup w^{\prime} \bullet 0\right\}$ $=\left\{\left(\left(x_{1}, y_{1} T, z_{1} I, t_{1} F\right),\left(x_{1}^{\prime}, y_{1}^{\prime} T, z_{1}^{\prime} I, t_{1}^{\prime} F\right)\right): x_{1} \in k \bullet a_{1}+k^{\prime} \bullet a_{2}\right.$,

$y_{1} \in k \bullet b_{1}+k^{\prime} \bullet b_{2} \cup m \bullet a_{1}+m^{\prime} \bullet a_{2} \cup m \bullet b_{1}+m^{\prime} \bullet b_{2}, z_{1} \in k \bullet c_{1}+k^{\prime} \bullet c_{2} \cup m \bullet c_{1}+m^{\prime} \bullet c_{2} \cup n \bullet a_{1}+$ $n^{\prime} \bullet a_{2} \cup n \bullet b_{1}+n^{\prime} \bullet b_{2} \cup n \bullet c_{1}+n^{\prime} \bullet c_{2}, t \in k \bullet d_{1}+k^{\prime} \bullet d_{2} \cup m \bullet d_{1}+m^{\prime} \bullet d_{2} \cup n \bullet d_{1}+n^{\prime} \bullet d_{2} \cup w \bullet a_{1}+$ $\left.w^{\prime} \bullet a_{2} \cup w \bullet b_{1}+w^{\prime} \bullet b_{2} \cup w \bullet c_{1}+w^{\prime} \bullet c_{2} \cup w \bullet d_{1}+w^{\prime} \bullet d_{2}, x_{1}^{\prime} \in 0, y_{1}^{\prime} \in 0, z_{1}^{\prime} \in 0, t_{1}^{\prime} \in 0\right\} \subseteq N(T, I, F)$. $\Longrightarrow \alpha \bullet u+\beta \bullet v \subseteq N(T, I, F)$.

Lastly, we can see from the definition of $N(T, I, F)$ that $N(T, I, F)$ contains a proper subset which is a hypervector space over $K$.

To this end we can conclude that $N(T, I, F)$ is a super strong NQ-Hypervector space.

Proposition 3.20. The intersection of any two

1. super strong $N Q$-Hypersubspaces of a super strong $N Q$-Hypervector space $V(T, I, F)$ over a neutrosophic quadruple field $(K, I, F)$ is again a super strong $N Q$-Hypersubspace of $V(T, I, F)$.

2. strong NQ-Hypersubspaces of a strong $N Q$-Hypervector space $V(T, I, F)$ over a neutrosophic field $K(I)$ is again a strong $N Q$-Hypersubspace of $V(T, I, F)$.

3. weak $N Q$-Hypersubspaces of a weak $N Q$-Hypervector space $V(T, I, F)$ over a field $K$ is again a weak $N Q$-Hypersubspace of $V(T, I, F)$.

Proof: Same as in classical case.

Proposition 3.21. Let $S(T, I, F)$ be a super strong $N Q$-Hypersubspace, $U(T, I, F)$ be a strong $N Q$-Hypersubspace and $W(T, I, F)$ be weak $N Q$-Hypersubspace of a super strong $N Q$-Hypervector space $(V(T, I, F),+, \bullet, K(T, I, F))$, strong $N Q$-Hypervector space $(V(T, I, F),+, \bullet, K(I))$ and weak $N Q$-Hypervector space $(V(T, I, F),+, \bullet, K)$ respectively. Then

1. $S(T, I, F) \cap U(T, I, F)$ is a strong $N Q$-Hypersubspace of strong $N Q$-Hypersubspace $(V(T, I, F),+, \bullet, K(I))$.

2. $S(T, I, F) \cap W(T, I, F)$ is a weak $N Q$-Hypersubspace of weak $N Q$-Hypersubspace $(V(T, I, F),+, \bullet, K)$.

3. $U(T, I, F) \cap W(T, I, F)$ is a weak $N Q$-Hypersubspace of weak $N Q$-Hypersubspace $(V(T, I, F),+, \bullet, K)$.

Proof:

1. By 1 of 3.7 we have that every super strong NQ-Hypervector space is a strong NQ-Hypervector space. Then by 2 of 3.20 the proof follows.

2. By applying 2 of 3.7 and 3 of 3.20 the proof follows easily.

3. By 3 of 3.7 we have that every strong NQ-Hypervector space is a weak NQ-Hypervector space. Then by applying 3 of 3.20 the proof follows.

Proposition 3.22. Let $U_{1}[T, I, F], U_{2}[T, I, F], \cdots, U_{n}[T, I, F]$ be NQ-Hypersubspace of a super strong[strong] NQ-Hypervector space $V(T, I, F)$ over a neutrosophic field $K(T, I, F)($ resp. $[K(I)])$. Then $\bigcap_{i=1}^{n} U_{i}$ is a $N Q$-Hypersubspace of $V(T, I, K)$.

Proof: Same as in classical case.

Example 3.23. Let $M_{1}[T, I, F]=K(T, I, F) \times\{(0,0 T, 0 I, 0 F)\} \subseteq V(T, I, F)$ and $M_{2}[T, I, F]=\{(0,0 T, 0 I, 0 F)\} \times K(T, I, F) \subseteq V(T, I, F)$.

Following the approach in Example 3.19 , we can establish that $M_{1}[T, I, F]$ and $M_{2}[T, I, F]$ are NQ-Hypersubspaces of $V(T, I, F)$.

Let $((a, b T, c I, d F),(0,0 T, 0 I, 0 F)) \in M_{1}[T, I, F]$ and $((0,0 T, 0 I, 0 F),(e, f T, g I, h F)) \in M_{2}[T, I, F]$. Then

$((a, b T, c I, d F),(0,0 T, 0 I, 0 F))+((0,0 T, 0 I, 0 F),(e, f T, g I, h F))=(((a+0),(b+0) T,(c+0) I,(d+$ 
$0) F),((0+e),(0+f) T,(0+g) I,(0+h) F))=((a, b T, c I, d F),(e, f T, g I, h F))$

But $\{((a, b T, c I, d F),(e, f T, g I, h F))\}$ is not a NQ-subset of $M_{1}[T, I, F] \cup M_{2}[T, I, F]$.

Therefore $M_{1}[T, I, F] \cup M_{2}[T, I, F]$ is not a NQ-Hypersubspace of $V(T, I, F)$.

This observation is recorded in the following remark.

Remark 3.24. Let $M_{1}[T, I, F]$ and $M_{2}[T, I, F]$ be NQ-Hypersubspaces of a super strong NQ-Hypervector space $V(T, I, F)$ over a NQ field $K(T, I, F)$, then generally, The union of two NQ-Hypersubspaces of a super strong NQ-Hypervector space $V(T, I, F)$ is not necessarily a NQ-Hypersubspace of $V(T, I, F)$.

Definition 3.25. Let $N_{1}[T, I, F]$ and $N_{2}[T, I, F]$ be any two NQ-Hypersubspaces of a super strong NQHypervector space $V(T, I, F)$ over a NQ field $K(T, I, F)$ then the sum of $N_{1}[T, I, F]$ and $N_{2}[T, I, F]$ denoted by $N_{1}[T, I, F]+N_{2}[T, I, F]$ is called NQ Hyperlinear sum or NQ linear sum of the NQ-Hypersubspaces $N_{1}[T, I, F]$ and $N_{2}[T, I, F]$. And it is defined by the set

$$
\bigcup\left\{n_{1}+n_{2}: n_{1}=\left(a_{1}, b_{1} T, c_{1} I, d_{1} F\right) \in N_{1}[T, I, F], n_{2}=\left(a_{2}, b_{2} T, c_{2} I, d_{2} F\right) \in N_{2}[T, I, F]\right\}
$$

The NQ Hyperlinear sum of $N_{1}[T, I, F]$ and $N_{2}[T, I, F]$ is called the direct sum of the NQ-Hypersubspaces $N_{1}[T, I, F]$ and $N_{2}[T, I, F]$ if $N_{1}[T, I, F] \cap N_{2}[T, I, F]=\{\theta\}$.

Proposition 3.26. Let $N_{1}[T, I, F]$ and $N_{2}[T, I, F]$ be any two $N Q$-Hypersubspaces of a super strong $N Q$ Hypervector space $V(T, I, F)$ over a $N Q$ field $K(T, I, F)$. Then

1. NQ Hyperlinear sum of $N_{1}[T, I, F]$ and $N_{2}[T, I, F]$ is a NQ-Hypersubspace of $V(T, I, F)$.

2. NQ Hyperlinear sum of $N_{1}[T, I, F]$ and $N_{2}[T, I, F]$ is the least $N Q$-Hypersubspace of $V(T, I, F)$ containing $N_{1}[T, I, F]$ and $N_{2}[T, I, F]$.

Proof:

1. Since $\theta=(0,0 T, 0 I, 0 F) \in N_{1}[T, I, F]$ and $\theta=(0,0 T, 0 I, 0 F) \in N_{2}[T, I, F]$, then $\{\theta+\theta\} \subseteq N_{1}[T, I, F]+N_{2}[T, I, F]$ $\Longrightarrow\{\theta\} \subseteq N_{1}[T, I, F]+N_{2}[T, I, F] \Longrightarrow \theta \in N_{1}[T, I, F]+N_{2}[T, F, I]$, therefore $N_{1}[T, I, F]+N_{2}[T, I, F]$ is non-empty. Let $u=(a, b T, c I, d F), v=(e, f T, g I, h F) \in N_{1}[T, I, F]+N_{2}[T, I, F]$, then $\exists$ $u_{1}=\left(a_{1}, b_{1} T, c_{1} I, d_{1} F\right), u_{2}=\left(a_{2}, b_{2} T, c_{2} I, d_{2} F\right) \in N_{1}\left[I_{1}, I_{2}\right]$ and $v_{1}=\left(e_{1}, f_{1} T, g_{1} I, h_{1} F\right)$ $v_{2}=\left(e_{1}, f_{1} T, g_{1} I, h_{1} F\right) \in N_{2}\left[I_{1}, I_{2}\right]$ such that $u \in u_{1}+v_{1}$ and $v \in u_{2}+v_{2}$.

Let $\alpha=(p, q T, r I, s F), \beta=\left(p^{\prime}, q^{\prime} T, r^{\prime} I, s^{\prime} F\right) \in K(T, I, F)$.

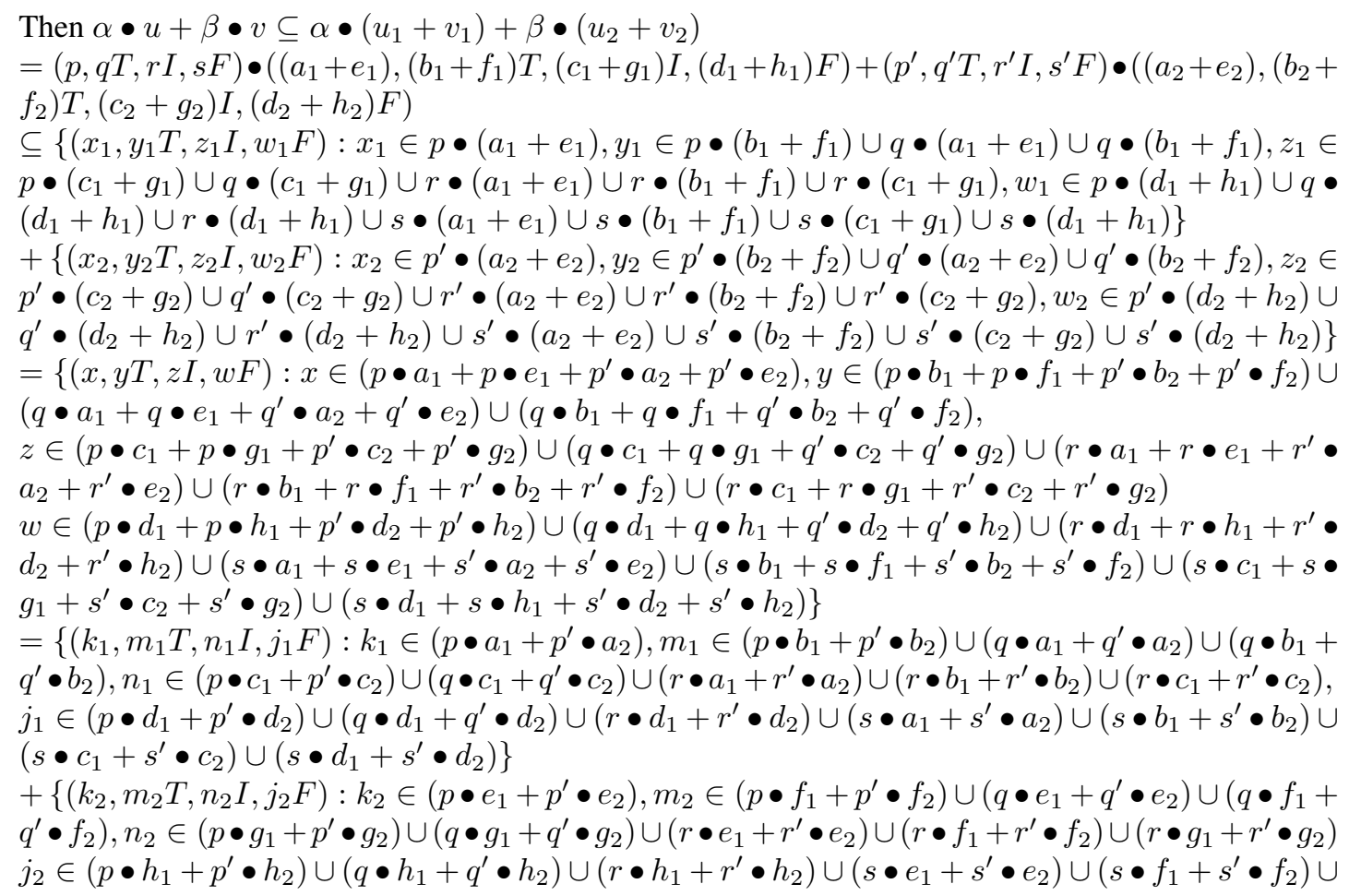


$\left.\left(s \bullet g_{1}+s^{\prime} \bullet g_{2}\right) \cup\left(s \bullet h_{1}+s^{\prime} \bullet h_{2}\right)\right\} \subseteq N_{1}[T, I, F]+N_{2}[T, I, F]$.

Hence $\alpha \bullet u+\beta \bullet v \subseteq N_{1}[T, I, F]+N_{2}[T, I, F]$.

Now since $N_{1}, N_{2}$ are proper subsets of $N_{1}[T, I, F]$ and $N_{2}[T, I, F]$ respectively, with both $N_{1}$ and $N_{2}$ being hypervector spaces. Then $N_{1}+N_{2}$ is a hypervector space which is properly contained in $N_{1}[T, I, F]+N_{2}[T, I, F]$. Then we can conclude that $N_{1}[T, I, F]+N_{2}[T, I, F]$ is a NQ-Hypersubspace.

2. Let $N[T, I, F]$ be NQ-Hypersubspace of $V(T, I, F)$ such that $N_{1}[T, I, F] \subseteq N[T, I, F]$ and $N_{2}[T, I, F] \subseteq N[T, I, F]$.

Let $u=(a, b T, c I, d F) \in N_{1}[T, I, F]+N_{2}[T, I, F]$, then $\exists u_{1}=\left(a_{1}, b_{1} T, c_{1} I, d_{2} F\right) \in N_{1}[T, I, F]$ and $u_{2}=\left(a_{2}, b_{2} T,, c_{2} I, d_{2} F\right) \in N_{2}[T, I, F]$ such that $u \in u_{1}+u_{2}$.

Since $N_{1}[T, I, F] \subseteq N[T, I, F]$ and $N_{2}[T, I, F] \subseteq N[T, I, F]$, then $u_{1}, u_{2} \in N[T, I, F]$.

Again since $N[T, I, F]$ is a NQ-Hypersubspace of $V(T, I, F)$, then we have that

$u_{1}+u_{2} \subseteq N[T, I, F] \Longrightarrow u \in N[T, I, F]$.

Hence $N_{1}[T, I, F]+N_{2}[T, I, F] \subseteq N[T, I, F]$ and the proof follows.

Proposition 3.27. Let $V(T, I, F)$ be a super strong $N Q$-Hypervector space over a $N Q$-field $K(T, I, F)$, let $u_{1}=\left(a_{1}, b_{1} T, c_{1} I, d_{1} F\right), u_{2}=\left(a_{2}, b_{2} T, c_{2} I, d_{2} F\right), \cdots, u_{n}=\left(a_{n}, b_{n} T, c_{n} I, d_{2} F\right) \in V(T, I, F)$ and $\alpha_{1}=\left(k_{1}, m_{1} T, r_{1} I, t_{1} F\right), \alpha_{2}=\left(k_{2}, m_{2} T, r_{2} I, t_{2} F\right) \cdots, \alpha_{n}=\left(k_{n}, m_{n} T, r_{n} I, t_{n} F\right) \in K(T, I, F)$. Then

1. $N(T, I, F)=\bigcup\left\{\alpha_{1} \bullet u_{1}+\alpha_{2} \bullet u_{2}+\cdots+\alpha_{n} \bullet u_{n}: \alpha_{1}, \alpha_{2}, \cdots, \alpha_{n} \in K(T, I, F)\right\}$ is a NQHypersubspace of $V(T, I, F)$.

2. $N(T, I, F)$ is the smallest NQ-Hypersubspace of $V(T, I, F)$ containing $u_{1}, u_{2}, \cdots, u_{n}$.

Proof:

1. Follow similar approach as that of proposition 3.26 above.

2. Suppose that $H(T, I, F)$ is a super strong NQ-Hypersubspace of $V(T, I, F)$ containing $u_{1}=\left(a_{1}, b_{1} T, c_{1} I, d_{1} F\right), u_{2}=\left(a_{2}, b_{2} T, c_{2} I, d_{2} F\right), \cdots, u_{n}=\left(a_{n}, b_{n} T, c_{n} I, d_{n} F\right)$.

Let $t \in N(T, I, F)$, then there exists $\alpha_{1}=\left(k_{1}, m_{1} T, p_{1} I, q_{1} F\right), \alpha_{2}=\left(k_{2}, m_{2} T, p_{2} I, q_{2} F\right), \cdots$, $\alpha_{n}=\left(k_{n}, m_{1} T, p_{1} I, q_{1} F\right) \in K(T, I, F)$ such that

$t \in \alpha_{1} \bullet\left(a_{1}, b_{1} T, c_{1} I, d_{1} F\right)+\alpha_{2} \bullet\left(a_{2}, b_{2} T, c_{2} I, d_{2} F\right)+\cdots+\alpha_{n} \bullet\left(a_{n}, b_{n} T, c_{n} I, d_{n} F\right) \subseteq H(T, I, F)$

Therefore $t \in H(T, I, F) \Longrightarrow N(T, I, F) \subseteq H(T, I, F)$.

Hence $N(T, I, F)$ is the smallest NQ-Hypersubspace of $V(T, I, F)$ containing $u_{1}, u_{2}, \cdots, u_{n}$.

Note: The NQ-Hypersubspace $N(T, I, F)$ of the super strong NQ-Hypervector space $V(T, I, F)$ over a NQ field $K(T, I, F)$ of proposition 3.27 is said to be generated or spanned by the NQ-Hypervectors $u_{1}, u_{2}, \cdots, u_{n}$ and we write $N(T, I, F)=\operatorname{span}\left\{u_{1}, u_{2}, \cdots, u_{n}\right\}$.

Definition 3.28. Let $N_{1}[T, I, F]$ and $N_{2}[T, I, F]$ be two NQ-Hypersubspaces of a super strong NQ-Hypervector space $(V(T, I, F),+, \bullet, K(T, I, F))$ over a NQ field $K(T, I, F) . V(T, I, F)$ is said to be the direct sum of $N_{1}[T, I, F]$ and $N_{2}[T, I, F]$ written $V(T, I, F)=N_{1}[T, I, F] \oplus N_{2}[T, I, F]$ if every element $v \in V(T, I, F)$ can be written uniquely as $v=n_{1}+n_{2}$ where $n_{1} \in N_{1}[T, I, F]$ and $n_{2} \in N_{2}[T, I, F]$.

Proposition 3.29. Let $N_{1}[T, I, F]$ and $N_{2}[T, I, F]$ be two NQ-Hypersubspaces of a super strong NQ-Hypervector space $(V(T, I, F),+, \bullet, K(T, I, F))$ over a $N Q$ field $K(T, I, F) . V(T, I, F)=N_{1}[T, I, F] \oplus N_{2}[T, I, F]$ if and only if the following conditions hold:

1. $V(T, I, F)=N_{1}[T, I, F]+N_{2}[T, I, F]$.

2. $N_{1}[T, I, F] \cap N_{2}[T, I, F]=\{\theta\}$.

Proof : Same as in classical case.

Example 3.30. Let $V(T, I, F)=\mathbb{R}^{3}(T, I, F)$ be a super strong NQ-Hypervector space over a NQ-field $R(T, I, F)$ and let

$N_{1}(T, I, F)=\{(u, \theta, w): u=(a, b T, c I, d F), w=(k, m T, n I, p F) \in R(T, I, F)\}$ and

$N_{2}(T, I, F)=\{(\theta, v, \theta): v=(e, f T, g I, h F) \in R(T, I, F)\}$, be super strong NQ-Hypersubspaces of $V(T, I, F)$. Then $V(T, I, F)=N_{1}(T, I, F) \oplus N_{2}(T, I, F)$. 
To see this, let $x=(u, v, w) \in V(T, I, F)$, then $x=(u, \theta, w)+(\theta, v, \theta)$, so $x \in N_{1}(T, I, F)+N_{2}(T, I, F)$. Hence $V(T, I, F)=N_{1}(T, I, F)+N_{2}(T, I, F)$.

To show that $N_{1}(T, I, F) \cap N_{2}(T, I, F)=\{\theta\}$, let $x=(u, v, w) \in N_{1}(T, I, F) \cap N_{2}(T, I, F)$.

Then $v=\theta$, i.e $(e, f T, g I, h F)=(0,0 T, 0 I, 0 F)$ because $\mathrm{x}$ lies in $N_{1}(T, I, F)$, and $u=w=\theta$ i.e $(a, b T, c I, d F)=(k, m T, n I, p F)=(0,0 T, 0 I, 0 F)$ because $\mathrm{x}$ lies in $N_{2}(T, I, F)$. Thus $x=(\theta, \theta, \theta)=\theta$, so $\theta=(0,0 T, 0 I, 0 F)$ is the only NQ-Hypervector in $N_{1}(T, I, F) \cap N_{2}(T, I, F)$.

Hence $N_{1}(T, I, F) \cap N_{2}(T, I, F)=\{0,0 T, 0 I, 0 F\}=\{\theta\}$.

$\Longrightarrow V(T, I, F)=N_{1}(T, I, F) \oplus N_{2}(T, I, F)$.

Definition 3.31. Let $N[T, I, F]$ be a NQ-Hypersubspace of a super strong NQ-Hypervector space

$(V(T, I, F),+, \bullet, K(T, I, F))$ over a NQ-field $K(T, I, F)$. The quotient $V(T, I, F) / N[T, I, F]$ is defined by the set

$$
\{[v]=v+N[T, I, F]: v \in V(T, I, F)\} .
$$

If for every $[u],[v] \in V(T, I, F) / N[T, I, F]$ and $\alpha \in K(T, I, F)$, we define:

$$
[u] \oplus[v]=(u+v)+N[T, I, F]
$$

and

$$
\alpha \odot[u]=[\alpha \bullet u]=\{[x]: x \in \alpha \bullet u\},
$$

it can be shown that $(V(T, I, F) / N[T, I, F], \oplus, \odot, K(T, I, F))$ is a super strong NQ-Hypervector space over NQ-field $K(T, I, F)$ called a super strong NQ quotient hypervector space.

\section{Linear Dependence, Independence, Bases and Dimensions of NQ- Hypervector Space}

Definition 4.1. Let $(V(T, I, F),+, \bullet, K(T, I, F))$ be a super strong NQ-Hypervector space over a NQ field $K(T, I, F)$ and let $B(T, I, F)=\left\{u_{1}=\left(a_{1}, b_{1} T, c_{1} I, d_{1} F\right), u_{2}=\left(a_{2}, b_{2} T, c_{2} I, d_{2} F\right), \cdots, u_{n}=\left(a_{n}, b_{n} T, c_{n} I, d_{n} F\right)\right\}$ be a subset of $V(T, I, F) . B(T, I, F)$ is said to generate or span $V(T, I, F)$ if $V(T, I, F)=\operatorname{span}(B(T, I, F))$.

Example 4.2. Let $V(T, I, F)=\mathbb{R}^{4}(T, I, K)$ be a super strong NQ-Hypervector space over a NQ field $R(T, I, F)$ and let $B(T, I, F)=\left\{u_{1}=((1,0 T, 0 I, 0 F),(0,0 T, 0 I, 0 F),(0,0 T, 0 I, 0 F),(0,0 T, 0 I, 0 F))\right.$, $u_{2}=((0,0 T, 0 I, 0 F),(1,0 T, 0 I, 0 F),(0,0 T, 0 I, 0 F),(0,0 T, 0 I, 0 F))$,

$u_{3}=((0,0 T, 0 I, 0 F),(0,0 T, 0 I, 0 F),(1,0 T, 0 I, 0 F),(0,0 T, 0 I, 0 F))$,

$\left.u_{4}=((0,0 T, 0 I, 0 F),(0,0 T, 0 I, 0 F),(0,0 T, 0 I, 0 F),(1,0 T, 0 I, 0 F))\right\}$.

Then $B(T, I, F)$ spans $V(T, I, F)$.

Definition 4.3. Let $(V(T, I, F),+, \bullet, K(T, I, F))$ be a super strong NQ-Hypervector space over NQ-field $K(T, I, F)$. The NQ vector $u=(a, b T, c I, d F) \in V(T, I, F)$ is said to be a linear combination of the NQ vectors $u_{1}=\left(a_{1}, b_{1} T, c_{1} I, d_{1} F\right), u_{2}=\left(a_{2}, b_{2} T, c_{2} I, d_{2} F\right), \cdots, u_{n}=\left(a_{n}, b_{n} T, c_{n} I, d_{n} F\right) \in V\left(I_{1}, I_{2}\right)$ if there exists NQ-scalars $\alpha_{1}=\left(k_{1}, m_{1} T, s_{1} I, t_{1} F\right), \alpha_{2}=\left(k_{2}, m_{2} T, s_{2} I, t_{2} F\right), \cdots, \alpha_{n}=\left(k_{n}, m_{n} T, s_{n} I, t_{n} F\right) \in$ $K(T, I, F)$ such that

$$
u \in \alpha_{1} \bullet u_{1}+\alpha_{2} \bullet u_{2}+\cdots+\alpha_{n} \bullet u_{n} .
$$

Example 4.4. Let $V(T, I, F)=\mathbb{R}(T, I, F)$ be a weak NQ-Hypervector space over a field $K=\mathbb{R}$. An element $v=(1,1 T, 4 I, 7 F) \in V(T, I, F)$ is a linear combination of the elements $v_{1}=(1,2 T,-1 I,-2 F)$, $v_{2}=(3,5 T, 2 I, 3 F) \in V(T, I, F)$

Since

$$
(1,1 T, 4 I, 7 F) \in-2 \bullet(1,2 T,-1 I,-2 F)+1 \bullet(3,5 T, 2 I, 3 F) .
$$

Definition 4.5. Let $(V(T, I, F),+, \bullet, K(T, I, F))$ be a super strong NQ-Hypervector space over a NQ field $K(T, I, F)$ and let $B(T, I, F)=\left\{u_{1}=\left(a_{1}, b_{1} T, c_{1} I, d_{1} F\right), u_{2}=\left(a_{2}, b_{2} T, c_{2} I, d_{2} F\right), \cdots, u_{n}=\left(a_{n}, b_{n} T, c_{n} I, d_{n} F\right)\right\}$ be a subset of $V(T, I, F)$.

1. $B(T, I, F)$ is called a linearly dependent set if there exists NQ scalars $\alpha_{1}=\left(k_{1}, m_{1} T, s_{1} I, t_{1} F\right), \alpha_{2}=$ $\left(k_{2}, m_{2} T, s_{2} I, t_{2} F\right), \cdots, \alpha_{n}=\left(k_{n}, m_{n} T, s_{n} I, t_{n} F\right)$ (not all zero) such that

$$
\theta \in \alpha_{1} \bullet u_{1}+\alpha_{2} \bullet u_{2}+\cdots+\alpha_{n} \bullet u_{n} .
$$


2. $B(T, I, F)$ is called a linearly independent set if

$$
\theta \in \alpha_{1} \bullet u_{1}+\alpha_{2} \bullet u_{2}+\cdots+\alpha_{n} \bullet u_{n}
$$

implies that $\alpha_{1}=\alpha_{2}=\cdots=\alpha_{n}=(0,0 T, 0 I, 0 F)=\theta$

Example 4.6. Let $V(T, I, F)=\mathbb{R}(T, I, F)$ be a weak NQ-Hypervector space over a field $K=\mathbb{R}$. The subset $B(T, I, F)=\{(5,-7 T, 5 I, 4 F),(3,-4 T, 2 I, 2 F),(-2,3 T,-3 T,-2 T)\}$ of $V(T, I, F)$ is NQ linearly dependent set since

$$
\theta \in 1 \bullet(5,-7 T, 5 I, 4 F)+(-1) \bullet(3,-4 T, 2 I, 2 F)+1 \bullet(-2,3 T,-3 T,-2 T)
$$

Example 4.7. Let $V(T, I, F)=\mathbb{R}(T, I, F)$ be a weak NQ-Hypervector space over a field $K=\mathbb{R}$. The subset $B(T, I, F)=\{(7,0 T, 0 I, 0 F),(0,3 T, 5 I, 0 F),(0,0 T, 0 T,-8 T)\}$ of $V(T, I, F)$ is NQ linearly independent set over $\mathbb{R}$ because we can not find $a, b, c \in \mathbb{R}$ such that

$$
\theta \in a \bullet(7,0 T, 0 I, 0 F)+b \bullet(0,3 T, 5 I, 0 F)+c \bullet(0,0 T, 0 T,-8 T)
$$

If possible then $\theta \in a \bullet(7,0 T, 0 I, 0 F)+b \bullet(0,3 T, 5 I, 0 F)+c \bullet(0,0 T, 0 T,-8 T)$ implies that;

$0 \in a \bullet 7+b \bullet 0+c \bullet 0$ which forces $a=0$,

$0 \in a \bullet 0+b \bullet 3+c \bullet 0$ which forces $b=0$,

$0 \in a \bullet 0+b \bullet 5+c \bullet 0$ which forces $b=0$ and

$0 \in a \bullet 0+b \bullet 0+c \bullet-8$ which forces $c=0$.

Thus the equations are consistent and $a=b=c=0$.

Proposition 4.8. Let $(V(T, I, F),+, \bullet, K)$ be a weak $N Q$-Hypervector space over a field $K$. Any singleton set of non-null $N Q$ vector of the weak $N Q$-Hypervector space $V(T, I, F)$ is linearly independent.

Proof: Suppose that $\theta \neq v=(a, b T, c I, d F) \in V(T, I, F)$. Let $\theta \in k \bullet v$ and suppose that $\theta \neq k \in K$. Then $k^{-1} \in K$ and therefore, $k^{-1} \bullet \theta \subseteq k^{-1} \bullet(k \bullet v)$ so that

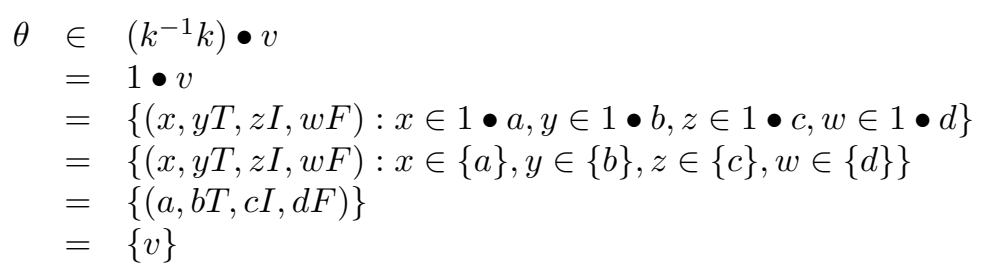

This shows that $v=\theta$ which is a contradiction. Hence, $k=\theta$ and thus, the singleton $\{v\}$ is a linearly independent set.

We note that the singleton set will be linearly dependent if it contains a null NQ-vector and $\theta \neq k \in K$. This observation is recorded in the next proposition.

Proposition 4.9. Let $(V(T, I, F),+, \bullet, K)$ be a weak $N Q$-Hypervector space over a field $K$. Any set of $N Q$ vectors of the weak $N Q$-Hypervector space $V(T, I, F)$ containing the null $N Q$-vector is always linearly dependent.

Proof: Follows from Proposition 4.8

Proposition 4.10. Let $(V(T, I, F),+, \bullet, K)$ be a weak $N Q$-Hypervector space over a field $K$ and let

$$
B\left(I_{1}, I_{2}\right)=\left\{u_{1}=\left(a_{1}, b_{1} T, c_{1} I, d_{1} F\right), u_{2}=\left(a_{2}, b_{2} T, c_{2} I, d_{2} F\right), \cdots, u_{n}=\left(a_{n}, b_{n} T, c_{n} I, d_{n} F\right)\right\}
$$

be a subset of $V(T, I, F)$. Then $B(T, I, F)$ is a linearly dependent set if and only if at least one element of $B(T, I, F)$ can be expressed as a linear combination of the remaining elements of $B(T, I, F)$.

Proof : Suppose that $B(T, I, F)$ is a linearly dependent set. Then there exists scalars $k_{1}, k_{2}, \cdots, k_{n}$ not all zero in $K$ such that

$$
\theta \in k_{1} \bullet u_{1}+k_{2} \bullet u_{2}+\cdots+k_{n} \bullet u_{n} .
$$

Suppose that $k_{1} \neq 0$, then $k_{1}^{-1} \in K$ and therefore

$$
\begin{aligned}
k_{1}^{-1} \bullet \theta & \subseteq k_{1}^{-1} \bullet\left(k_{1} \bullet u_{1}+k_{2} \bullet u_{2}+\cdots+k_{n} \bullet u_{n}\right) \\
& =\left(k_{1}^{-1} k_{1}\right) \bullet u_{1}+\left(k_{2}^{-1} k_{2}\right) \bullet u_{2}+\cdots+\left(k_{n}^{-1} k_{n}\right) \bullet u_{n} \\
& =1 \bullet u_{1}+\left(k_{1}^{-1} k_{2}\right) \bullet u_{2}+\cdots+\left(k_{1}^{-1} k_{n}\right) \bullet u_{n}
\end{aligned}
$$


This implies that

$$
\begin{aligned}
-u_{1} & \in\left(k_{1}^{-1} k_{2}\right) \bullet u_{2}+\cdots+\left(k_{1}^{-1} k_{n}\right) \bullet u_{n} \\
\left(u_{1}\right) & \in(-1) \bullet\left[\left(k_{1}^{-1} k_{2}\right) \bullet u_{2}+\cdots+\left(k_{1}^{-1} k_{n}\right) \bullet u_{n}\right] \\
& \subseteq(-1) \bullet\left(\left(k_{1}^{-1} k_{2}\right) \bullet u_{2}+\cdots+(-1) \bullet\left(k_{n}^{-1} k_{n}\right) \bullet u_{n}\right) \\
& \subseteq\left(-k_{1}^{-1} k_{2}\right) \bullet u_{2}+\left(-k_{1}^{-1} k_{3}\right) \bullet u_{3}+\cdots+\left(-k_{1}^{-1} k_{n}\right) \bullet u_{n} .
\end{aligned}
$$

This shows that $u_{1} \in \operatorname{span}\left\{u_{2}, u_{3}, \cdots, u_{n}\right\}$.

Conversely, suppose that $u_{1} \in \operatorname{span}\left\{u_{2}, u_{3}, \cdots, u_{n}\right\}$ and suppose that $0 \neq-1 \in K$. Then there exists $k_{2}, k_{3}, \cdots, k_{n} \in K$ such that

$$
u_{1} \in k_{2} \bullet u_{2}+k_{3} \bullet u_{3}+\cdots+k_{n} \bullet u_{n}
$$

and we have

$$
u_{1}+\left(-u_{1}\right) \in(-1) \bullet u_{1}+k_{2} \bullet u_{2}+k_{3} \bullet u_{3}+\bullet+k_{n} \bullet u_{n} .
$$

from which we have

$$
\theta \in(-1) \bullet u_{1}+k_{2} \bullet u_{2}+k_{3} \bullet u_{3}+\cdots+k_{n} \bullet u_{n} .
$$

Since $-1 \neq 0 \in K$, it follows that $B(T, I, F)$ is a linearly dependent set.

Proposition 4.11. Let $(V(T, I, F),+, \bullet, K(T, I, F))$ be a super strong $N Q$-Hypervector space over a $N Q$ field $K(T, I, F)$ and let $M(T, I, F)$ and $N(T, I, F)$ be subsets of $V(T, I, F)$ such that $M(T, I, F) \subseteq N(T, I, F)$.

1. If $M(T, I, F)$ is linearly dependent, then $N(T, I, F)$ is linearly dependent.

2. If $N(T, I, F)$ is linearly independent, then $M(T, I, F)$ is linearly independent.

Proof: Same as in classical case.

Definition 4.12. Let $V(T, I, F)$ be a super strong(strong) NQ-Hypervector space over a NQ field $K(T, I, F)$ (resp. neutrosophic field $K(I)$ ) and let $B(T, I, F)=\left\{u_{1}=\left(a_{1}, b_{1} T, c_{1} I, d_{1} F\right), u_{2}=\left(a_{2}, b_{2} T, c_{2} I, d_{2} F\right), \cdots, u_{n}=\left(a_{n}, b_{n} T, c_{n} I, d_{n} F\right)\right\}$ be a subset of $V(T, I, F) . B(T, I, F)$ is said to be a basis for $V(T, I, F)$ if the following conditions hold:

1. $B(T, I, F)$ is a linearly independent set

2. $V(T, I, F)=\operatorname{span}(B(T, I, F))$.

If $B(T, I, F)$ is finite and its cardinality is $\mathrm{n}$, then $V(T, I, F)$ is called an n-dimensional super strong(strong) NQ-Hypervector space and we write $\operatorname{dim}_{s s}(V(T, I, F))\left(\operatorname{resp} .\left(\operatorname{dim}_{s} V(T, I, F)\right)\right)=n$. If $B(T, I, F)$ is not finite, then $V(T, I, F)$ is called an infinite-dimensional super strong(strong) NQ-Hypervector space.

Example 4.13. In 4.2 $B(T, I, F)$ is a basis for $V(T, I, F)$ and $\operatorname{dim}_{s s} V(T, I, F)=4$

Proposition 4.14. Let $(V(T, I, F),+, \bullet, K(T, I, F))$ be a finite dimensional super strong NQ-Hypervector space over a $N Q$ field $K(T, I, F)$ and let

$B(T, I, F)=\left\{x_{1}=\left(a_{1}, b_{1} T, c_{1} I, d_{1} F\right), x_{2}=\left(a_{2}, b_{2} T, c_{2} I, d_{2} F\right), \cdots, u_{n}=x_{n}=\left(a_{n}, b_{n} T, c_{n} I, d_{n} F\right)\right\}$ be a basis for $V(T, I, F)$. Then every non null $N Q$-Hypevector $x=(a, b T, c I, d F) \in V(T, I, K)$ has a unique representation.

Proof: Since $B(T, I, F)$ is a basis for $V(T, I, F)$ and $x \in V(T, I, K)$, there exist $\alpha_{1}=\left(k_{1}, m_{1} T, n_{1} I, t_{1} F\right)$, $\alpha_{2}=\left(k_{2}, m_{2} T, n_{2} I, t_{2} F\right), \cdots, \alpha_{n}=\left(k_{n}, m_{n} T, n_{n} I, t_{n} F\right)$ such that

$$
x \in \alpha_{1} \bullet x_{1}+\alpha_{2} \bullet x_{2}+\cdots+\alpha_{n} \bullet x_{n}
$$

Suppose we also let $x \in \beta_{1} \bullet x_{1}+\beta_{2} \bullet x_{2}+\cdots+\beta_{n} \bullet x_{n}$, for some $\beta_{1}=\left(p_{1}, q_{1} T, r_{1} I, s_{1} F\right)$, $\beta_{2}=\left(p_{2}, q_{2} T, r_{2} I, s_{2} F\right), \cdots, \beta_{n}=\left(p_{n}, q_{n} T, r_{n} I, s_{n} F\right) \in K(T, I, F)$.

Therefore, $-x \in(-1) \bullet x \subseteq(-1) \bullet\left(\beta_{1} \bullet x_{1}+\beta_{2} \bullet x_{2}+\cdots+\beta_{n} \bullet x_{n}\right)$

$$
\begin{array}{lll}
\Longrightarrow & -x \in\left((-1) \bullet\left(\beta_{1} \bullet x_{1}\right)\right)+\left((-1) \bullet\left(\beta_{2} \bullet x_{2}\right)\right)+\cdots+\left((-1) \bullet\left(\beta_{n} \bullet x_{n}\right)\right) \\
= & \left.\left(\left(-1 \bullet \beta_{1}\right) \bullet x_{1}\right)+\left(\left(-1 \bullet \beta_{2}\right) \bullet x_{2}\right)+\cdots+\left(\left(-1 \bullet \beta_{n}\right) \bullet x_{n}\right)\right) \\
= & \left(-\beta_{1}\right) \bullet x_{1}+\left(-\beta_{2}\right) \bullet x_{2}+\cdots+\left(-\beta_{n}\right) \bullet x_{n} .
\end{array}
$$

Therefore

$$
-x \in\left(-\beta_{1}\right) \bullet x_{1}+\left(-\beta_{2}\right) \bullet x_{2}+\cdots+\left(-\beta_{n}\right) \bullet x_{n}
$$


From (1) and (2) we obtain

$$
x+(-x) \subseteq\left(\alpha_{1} \bullet x_{1}+\alpha_{2} \bullet x_{2}+\cdots+\alpha_{n} \bullet x_{n}\right)+\left(\left(-\beta_{1}\right) \bullet x_{1}+\left(-\beta_{2}\right) \bullet x_{2}+\cdots+\left(-\beta_{n}\right) \bullet x_{n}\right) .
$$

Therefore $\theta \in x+(-x) \subseteq\left(\alpha_{1}+\left(-\beta_{1}\right)\right) \bullet x_{1}+\left(\alpha_{2}+\left(-\beta_{2}\right)\right) \bullet x_{2}+\cdots+\left(\alpha_{n}+\left(-\beta_{n}\right)\right) \bullet x_{n}$.

Since $\left\{x_{1}, x_{2}, \cdots, x_{n}\right\}$ is a basis for $V(T, I, F)$ and

$$
\theta \in\left(\alpha_{1}-\beta_{1}\right) \bullet x_{1}+\left(\alpha_{2}-\beta_{2}\right) \bullet x_{2}+\cdots+\left(\alpha_{n}-\beta_{n}\right) \bullet x_{n} .
$$

Then it follows that $\theta \in \alpha_{i}-\beta_{i}$, for all $i=1,2, \cdots, n$. Hence $a_{i}=b_{i}$, for all $i=1,2, \cdots, n$.

\section{Conclusion}

In this paper, we have studied Hypervector Space in the Neutrosophic Quadruple (NQ) environment. Their basic properties have been extended and established in the Neutrosophic Quadruple (NQ) environment. We hope to study the homomorphsms and establish more advanced properties of this structure in our future work.

\section{References}

[1] Agboola, A.A.A. and Akinleye, S.A., Neutrosophic Hypervector Spaces, ROMAI Journal, vol. 11(1), pp. $1-16,2015$.

[2] Agboola, A.A.A. and Akinleye, S.A., Neutrosophic Vector Spaces, Neutrosophic Set and Systems, vol. 4, pp. 9-18, 2012.

[3] Agboola, A.A.A., Davvaz, B., Smarandache, F. Neutrosophic quadruple algebraic hyperstructures. Ann.Fuzzy Math. Inform. vol. 14, pp. 29-42. 2017.

[4] Akinleye, S.A., Smarandache, F., Agboola, A.A.A. On neutrosophic quadruple algebraic structures. Neutrosophic Sets System. vol. 12, pp. 122-126, 2016.

[5] Ameri, R. Fuzzy hypervector spaces over valued fields, Iranian Journal of Fuzzy Systems.vol. 2, pp. 37-42, 2005.

[6] Ameri, R. Fuzzy (co-) norm hypervector spaces, Proceeding of the 8th International Congress in Algebraic Hyperstructures and Applications,Samotraki, Greece, pp.71-79, 2002.

[7] Ameri, R., Dehghan,O.R., On dimension of hypervector spaces, European Journal of Pure and Applied Mathematics, Vol. 1(2),pp. 32-50, 2008.

[8] Ameri, R., Dehghan,O.R., Fuzzy hypervector spaces, Advances in Fuzzy Systems, Article ID 295649, 2008.

[9] Broumi, S., Bakali, A., Talea, M., Smarandache, F.,Singh, P. K., Uluçay, V., and Khan, M., Bipolar complex neutrosophic sets and its application in decision making problem. In Fuzzy Multi-criteria DecisionMaking Using Neutrosophic Sets. Springer, Cham. pp. 677-710, 2019.

[10] Ibrahim M.A, Agboola,A.A.A, Adeleke, E.O. and Akinleye, S.A, Introduction to Neutrosophic Subtraction Algebra and Neutrosophic Subtraction Semigroup, International Journal of Neutrosophic Science, vol.2, pp. 47-62, 2020.

[11] Jun, Y.B., Song, S.Z., Kim, S.J. Neutrosophic Quadruple BCI-Positive Implicative Ideals. Mathematics vol. 7, pp. 385, 2019.

[12] Jun,Y., Song,S.Z., Smarandache,F., Bordbar, H., Neutrosophic quadruple BCK/BCI-algebras. Axioms, vol.7, pp. 41, 2018.

[13] Krasner,M. A class of hyperrings and hyperfields, Intern. J.Math. and Math. Sci., Vol 6, no.2, pp. 307312, 1983.

[14] Li, Q., Ma, Y., Zhang, X., Zhang, J. Neutrosophic Extended Triplet Group Based on Neutrosophic Quadruple Numbers. Symmetry, vol. 11, pp. 696. 2019. 
[15] Madeleine,Al-Tahan. Some Result on Single Valued Neutrosophic (Weak) Polygroups. International Journal of Neutrosophic Science, vol.2, pp. 38-46, 2020.

[16] Marty,F., Sur une generalisation de la notion de groupe., In 8'eme congr'es des Mathematiciens Scandinaves, Stockholm, pp. 45-49, 1934.

[17] Muhiuddin, G., Al-Kenani, A.N., Roh, E.H., Jun, Y.B. Implicative Neutrosophic Quadruple BCKAlgebras and Ideals. Symmetry, vol. 11,pp. 277, 2019.

[18] Muhiuddin, G. Smarandache, F., Young Bae Jun, Neutrosophic quadruple ideals in neutrosophic quadruple BCI-algebras. Neutrosophic Sets and Systems, Vol.25,pp.161-173, 2019.

[19] Şahin, M. and Kargın A., Neutrosophic triplet groups based on set valued neutrosophic quadruple numbers, Neutrosophic Set and Systems, vol. 30, pp. $122-131,2019$.

[20] Şahin M. and Kargın A., Neutrosophic Triplet v-Generalized Metric Space, Axioms- MDPI, vol.7, pp. 67-73, 2018.

[21] Şahin, M. and Kargın A., Single valued neutrosophic quadruple graphs, Asian Journal of Mathematics and Computer Research, vol. 26(4), pp. 243-250, 2019.

[22] Şahin M., Kargın A. and Çoban M. A., Fixed point theorem for neutrosophic triplet partial metric space, Symmetry- MDPI, vol.10(7), pp. 240-246, 2018.

[23] Sanjay, R. Samanta, T.K., A Note on Hypervector Spaces. arXiv:1002.3816v3[math.GM], pp.16, 2011.

[24] Scafati Tallini. M., Matroidal Hypervector spaces, Journal of Geometry, vol. 42, pp.132-140, 1991.

[25] Scafati Tallini, M., Weak hypervector spaces and norms in such spaces, Proceeding of International Congress in Algebraic Hyperstructures and Applications, Iasi, Romania, Luglio(1993), Hadronic Press, Palm Harbor, Florida (U.S.A.), pp. 109-206. 1994.

[26] Scafati Tallini,M. Characterization of Remarkable Hypervector spaces, Proceedings of 8th international Congress on Algebraic Hyperstructures and Applications, Samotraki, Greece, (Sept. 1-9 2002), Spanidis Press, Xanthi, Greece, ISBN 960-87499-5-6, pp. 231-237, 2003.

[27] Smarandache, F., A Unifying Field in Logics: Neutrosophic Logic, Neutrosophy, Neutrosophic Set, Neutrosophic Probability, American Research Press, Rehoboth, 2003.

[28] Smarandache, F., Introduction to Neutrosophic Statistics, Sitech and Education Publishing, Romania, 2014.

[29] Smarandache, F., Neutrosophic Probability, Set and Logic, Am Res Press, Rehoboth, USA, 1998.

[30] Smarandache, F., NeurtoAlgebra is a Generalization of Partial Algebra. International Journal of Neutrosophic Science, vol.2, pp. 08-17, 2020.

[31] Smarandache,F., Neutrosophic Quadruple Numbers, Refined Neutrosophic Quadruple Numbers, Absorbance Law,and the Multiplication of Neutrosophic Quadruple Numbers. Neutrosophic Sets and System. vol.10, pp. 96-98. 2015.

[32] Vasantha Kandasamy, W.B and Smarandsche,F., Neutrosophic Ring, Hexis Phoenix, Arizona 2006.

[33] Vansantha Kandasamy, W.B. and Smarandache,F., Some Neutrosophic Algebraic Structures and Neutrosophic N-Algebraic Structures, Hexis, Phoenix, Arizona, 2006.

[34] Vasantha Kandasamy W.B., Ilanthenral Kandasamy, Smarandache, F. Neutrosophic Quadruple Vector Spaces and Their Properties. Mathematics, vol.7, pp. 758-765, 2019. 